Vol. 5, No. 3-4, 2019

\author{
Andrij Andrukhiv ${ }^{1}$, Bohdan Sokil ${ }^{2}$, Mariia Sokil ${ }^{3}$ \\ 1. Scientific Library, Lviv Polytechnic National University, Ukraine, Lviv, Profesorska Street, 1, \\ E-mail: andriy.i.andrukhiv@1pnu.ua \\ 2. Department of Engineering Mechanics, Hetman Petro Sahaidachny National Army Academy, \\ Ukraine, Lviv, Heroes of Maidan Street, 32, E-mail: Bogdan.I.Sokil@lpnu.ua \\ 3. Department of Transport Technologies, Lviv Polytechnic National University, \\ Ukraine, Lviv, Stepan Bandera Street, 12, E-mail: Mariia.B.Sokil@lpnu.ua
}

\title{
WAVE CONCEPT OF MOTION IN MATHEMATICAL MODELS OF THE DYNAMICS OF TWO-DIMENSIONAL MEDIA STUDYING
}

Received: November 12, 2019 / Revised: December 21, 2019 / Accepted: Deember 28, 2019

(C) Andrukhiv A., Sokil B., Sokil M., 2019

\begin{abstract}
The methodology of the studying of dynamic processes in two-dimensional systems by mathematical models containing nonlinear equation of Klein-Gordon was developed. The methodology contains such underlying: the concept of the motion wave theory; the single frequency fluctuations principle in nonlinear systems; the asymptotic methods of nonlinear mechanics. The aggregate content allowed describing the dynamic process for the undisturbed (linear) analogue of the mathematical model of movement. The value determining the impact of nonlinear forces on the basic parameters of the waves for the disturbed analogue is defined.
\end{abstract}

Keywords: dispersion ratio, amplitude, frequency, wave number, mathematical model, nonlinear oscillations, asymptotic solution.

\section{Introduction}

The concept of wave motion in the study of various dynamic processes has been widely used for many linear models of physical or mechanical systems. However, in the linear models cannot always explain many phenomena that take place in real systems. First of all talking about the fluctuations of one and many-dimensional bodies and environments. The problem is much more complicated for the case when the last are characterized by constant or variable longitudinal movement speed [1], [2], [3], [4] (rope tensions, belt and chain gear, loose medium during vibratory processing, pipelines along which the fluid moves and others). It is impossible to apply the classical methods such as Fourier or D'Alembert for analytical studies even linear (simplified) oscillating models of these systems. Despite the specified, the systems classes are allocated in the work. Their mathematical models of the process are same type of nonlinear partial differential equations, which contains mixed derivative of linear and time variables. It takes into consideration the longitudinal component of the medium movement and associated with it major problems linear models of systems integrating. Based on the wave theory of motion the single - frequency decoupling is managed to build for it. This in turn has served the basis for consideration nonlinear models of systems, dissemination of asymptotic methods of nonlinear mechanics [5], [6], [7] on new classes of dynamical systems. They allowed receiving the calculated dependences to research the impact of physical - mechanical parameters, nonlinear forces and speed of longitudinal motion on the main characteristics of a dynamic process.

\section{Problem Statement}

As known the mathematical model of nonlinear longitudinal or transverse vibrations of twodimensional environment in Euler variables which characterized by a constant speed of longitudinal motion $V$ is differential equation:

$$
u_{t t}+2 V u_{x t}-\alpha^{2} u_{x x}-\beta^{2} u_{y y}+\gamma u=\varepsilon f\left(u, u_{x}, u_{y}, u_{t}, u_{x x}, u_{y y}, u_{x y}\right),
$$


where $\alpha, \beta, \gamma$ are constants, which are expressed through the physical and mechanical characteristics of the environment; $\varepsilon f\left(u, u_{x}, u_{y}, u_{t}, u_{x x}, u_{y y}, u_{x y}\right)$ is known analytic function; $\varepsilon$ is small parameter.

For equation (1) we consider the form of boundary conditions:

$$
u(t, x, y)_{\mid x=0}=u(t, x, y)_{\mid x=l}=0 .
$$

The problem is to determine the impact on environment entire spectrum of external and internal factors dynamical process. It is fully possible to make only based on the solution of the boundary value problem (1), (2). For its location the basic ideas presented in [8], [9] are developed in this paper. Their essence is to spread the concept of wave motion in case of longitudinally moving media.

It should be noted that, based on the wave theory of motion in the works [10], [11], asymptotic approximation quasilinear and nonlinear models of one-dimensional equations Klein-Gordon and Brezerton, that consider the dynamics of their environments without longitudinal movement are built. It is reviewed the cases of a so-called "long systems" for which the boundary conditions were not considered. In papers [5], [6] the basic idea is spread in case of "short systems" with consideration of appropriate boundary conditions. This shows: the environment dynamic process in the case of one-dimensional medium, which characterized by a constant velocity component longitudinal movement, can be seen as overlapping waves of different lengths but identical frequencies. Moreover, based on the above the asymptotic approximation aces for perturbed models of these equations were managed to built, autonomous and non autonomous case was reviewed.

\section{Mathematical model}

Considering that the right side of equation (1) is proportional to the small parameter $\varepsilon$ for constructing asymptotic solutions formulated above boundary value problem we can apply the general ideas of perturbation methods. According to the latter, we'll show that the unperturbed $(\varepsilon=0)$ boundary value problem that corresponds (1), (2), namely:

$$
\begin{gathered}
u_{0 t t}+2 V u_{0 x t}-\left(\alpha^{2}-V^{2}\right) u_{0 x x}-\beta^{2} u_{0 y y}-\gamma^{2} u_{0}=0 ; \\
u_{0}(t, x, y)_{\mid x=0}=u_{0}(t, x, y)_{\mid x=l}=0
\end{gathered}
$$

has a solution of the wave type. Developing the basic idea of works [8], [9], indeed the solution of equation (3) for the boundary conditions (4) will be sought in the form:

$$
u(t, x, y)=a \cos (\kappa x+\delta y+\omega t+\phi)+b \cos (\chi x-\delta y-\omega t+\psi),
$$

where, respectively $\omega$ is frequency; $a, b$ are amplitudes of the direct and reflected waves; $\kappa, \chi$ are their wave numbers; $\delta$ is a wave number of the transverse component of the wave; $\phi, \psi$ are initial phases of the waves.

Representing the solution in the form (5) doesn't contradict to known classical results, because of it, as will be shown below were obtained known classical value as a special case when $\gamma=\delta=0$. Indeed, substituting (3) in place of derivative expressions which deriving from (5), to contact between frequency and wavenumber the dispersion relations are obtained:

$$
\begin{aligned}
& \omega^{2}+2 V \omega \kappa-\left(\alpha^{2}-V^{2}\right) \kappa^{2}-\beta^{2} \delta^{2}-\gamma^{2}=0, \\
& \omega^{2}-2 V \omega \chi-\left(\alpha^{2}-V^{2}\right) \chi^{2}-\beta^{2} \delta^{2}-\gamma^{2}=0 .
\end{aligned}
$$

Also, the representation of the solution in the form (5) will satisfy the boundary condition at $x=0$, if there is a relationship between the amplitudes and initial phases of the wave form $a=-b \phi=-\psi$, Similarly the representation of the solution in the form (5) will satisfy the conditions in $x=l$, if the identity is performed:

$$
\cos (\kappa l+\delta y+\omega t+\phi)-\cos (\chi l-\delta y-\omega t-\phi) \equiv 0 .
$$

Identity (7) will be carried out at any moment of time $t$, if there is a connection between wave numbers $\kappa, \chi, \delta$ and parameter $l$ : 


$$
\begin{gathered}
\cos (\kappa l+\delta y)-\cos (\chi l-\delta y) \equiv 0, \\
\sin (\kappa l+\delta y)-\sin (\chi l-\delta y) \equiv 0 .
\end{gathered}
$$

Identities (8) are defined by wave numbers $\kappa, \chi$ in the form:

$$
\kappa+\chi=\frac{2 k \pi}{l}, k=1,2, \ldots
$$

The dispersion relation (5), together with the dependence (9) allows determining the wave numbers $\kappa, \chi$ and frequency of process $\omega$ :

$$
\begin{gathered}
\kappa=\frac{\pi k}{l}+\frac{V \sqrt{l^{2} \gamma^{2} \delta^{2}+\pi^{2} k^{2}\left(\alpha^{2}-V^{2}\right)}}{\alpha l \sqrt{\alpha^{2}-V^{2}}}, \\
\chi=\frac{\pi k}{l}-\frac{V \sqrt{l^{2} \gamma^{2} \delta^{2}+\pi^{2} k^{2}\left(\alpha^{2}-V^{2}\right)}}{\alpha l \sqrt{\alpha^{2}-V^{2}}} \\
\omega=\frac{1}{\alpha l} \sqrt{\alpha^{2}-V^{2}} \cdot \sqrt{l^{2} \gamma^{2} \delta^{2}+\pi^{2} k^{2}\left(\alpha^{2}-V^{2}\right)} .
\end{gathered}
$$

In the obtained dependences parameter $\delta$ is the wave number of transverse waves that remains undefined. It can be found with additional conditions. In particular assuming that across the medium fits integer number of half-wave, than the parameter $\delta$ takes the value $\delta=\frac{m \pi}{b}$ ( $b$ is the width environment, $m=1,2, \ldots)$. Thus, the specified assumptions the dynamic process in a linear environment model described by the relation:

$$
\begin{gathered}
u_{0}(t, x, y)=a \cos \left[\left(\left(\frac{k \pi}{l}+\frac{V}{\alpha} \Delta\right) x+\frac{m \pi}{b} y+\frac{\alpha^{2}-V^{2}}{\alpha} \Delta t+\phi\right)-\right. \\
\left.-\cos \left(\left(\frac{k \pi}{l}-\frac{V}{\alpha} \Delta\right) x-\frac{m \pi}{b} y-\frac{\alpha^{2}-V^{2}}{\alpha} \Delta t-\phi\right)\right] ; \\
\Delta=\frac{\sqrt{l^{2} \gamma^{2} \delta^{2}+\pi^{2} k^{2}\left(\alpha^{2}-V^{2}\right)}}{l \sqrt{\alpha^{2}-V^{2}}} .
\end{gathered}
$$

Submitted graphical dependencies show the impact of physical and mechanical properties and the speed of longitudinal movement on the dynamics of the linear model environment. In particular, for larger values of velocity of longitudinal movement the frequency of natural oscillations is smaller and at a speed of longitudinal motion $V=\alpha$ oscillation is failured.

The obtained value pave the way for the study of the effect on the dynamic process of nonlinear forces, that is, to derive the solution perturbed equation. According to the general principles of construction of solutions nonlinear differential equations with a small parameter in the right side, the solution perturbed equation (1) for homogeneous boundary conditions (2) will be sought in the form of asymptotic series:

$$
u(x, y, t)=a(\cos (\kappa x+\delta y+\theta)-\cos (\chi x-\delta y-\theta))+\varepsilon u_{1}(a, x, y, \theta)+\varepsilon^{2} u_{2}(a, x, y, \theta)+\ldots,
$$

where $\theta=\omega t+\phi$, and unknown functions $u_{1}(a, x, y, \theta), u_{2}(a, x, y, \theta), \ldots$ should be determined so that: a) asymptotic representation of the solution (12) satisfies with the required degree of accuracy the original equation (1); b) be periodic relatively to $x, y, \theta$; c) satisfy the boundary conditions arising from (2).

In addition, the nonlinear force is the reason that the parameters $a$ and $\phi$ for a perturbed case are variables. Below we consider the case when these parameters are depending on time, and do not change their values along the tape. Laws of changes will be sought in the form of differential relations:

$$
a_{t}=\varepsilon A_{1}(a)+\varepsilon^{2} A_{2}(a)+\ldots, \quad \phi_{t}=\varepsilon B_{1}(a)+\varepsilon^{2} B_{2}(a)+\ldots .
$$


Wave Concept of Motion in Mathematical Models of the Dynamics of Two-dimensional Media ...

Thereby, task is to identify these unknown functions $A_{1}(a), A_{2}(a), \ldots, B_{1}(a), B_{2}(a), \ldots$, and $u_{1}(a, x, y, \theta), \quad u_{2}(a, x, y, \theta), \ldots$, in which the asymptotic representation of the solution (11) with the necessary degree of accuracy satisfies the original equation (1). To do this by differentiating (12) with regard to the variable (13), we have:

$$
\begin{gathered}
u_{t}(x, t)=-a \omega[\sin (\kappa x+\delta y+\theta)+\sin ]+\varepsilon\left\{A_{1}(a)[\cos (\kappa x+\delta y+\theta)-\cos (\chi x-\delta y-\theta)]-\right. \\
\left.-a B_{1}(a)[\sin (\kappa x+\delta y+\theta)+\sin (\chi x-\delta y-\theta)]+\frac{\partial u_{1}}{\partial \theta} \omega\right\}+\varepsilon^{2} \ldots \\
u_{t t}(x, t)=-a \omega^{2}[\cos (\kappa x+\delta y+\theta)-\cos (\chi x-\delta y-\theta)]- \\
-\omega \varepsilon\left\{2 A_{1}(a)[\sin (\kappa x+\delta y+\theta)+\sin (\chi x-\delta y-\theta)]+\right. \\
\left.+2 a B_{1}(a)[\cos (\kappa x+\delta y+\theta)-\cos (\chi x-\delta y-\theta)]-\frac{\partial^{2} u_{1}}{\partial \theta^{2}} \omega\right\}+\varepsilon^{2} \ldots
\end{gathered}
$$

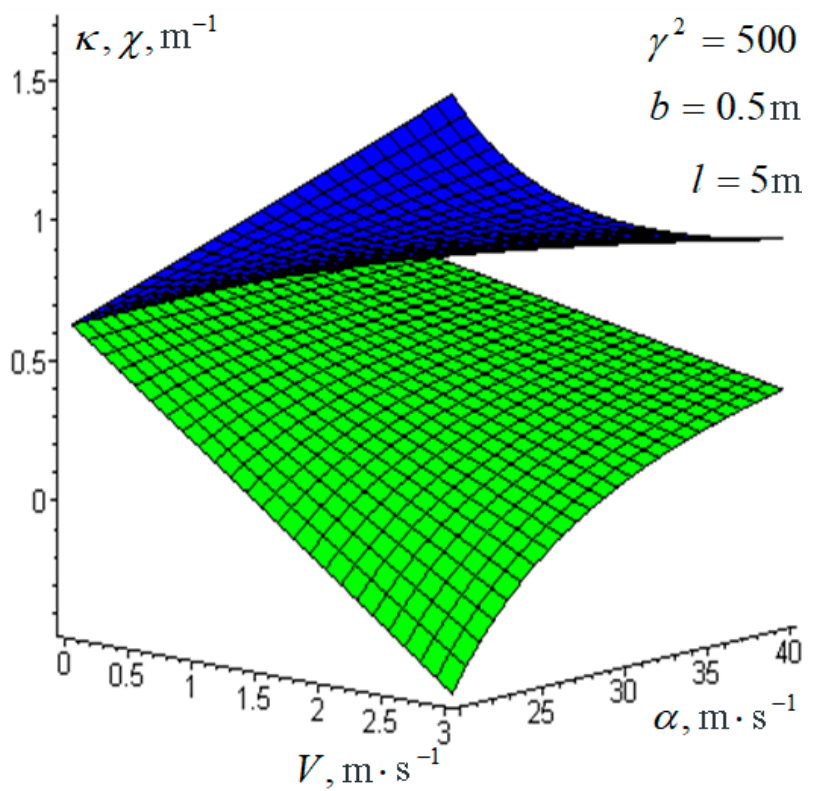

$\mathrm{a}$

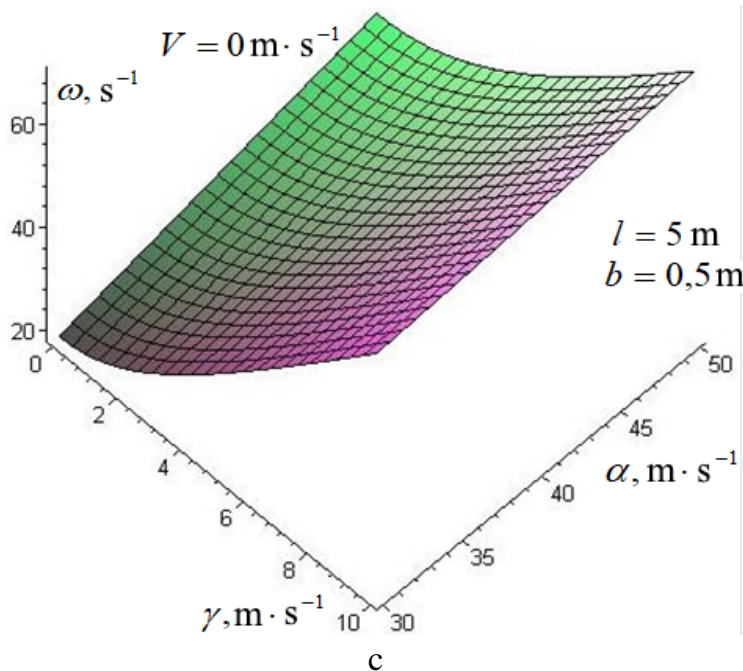

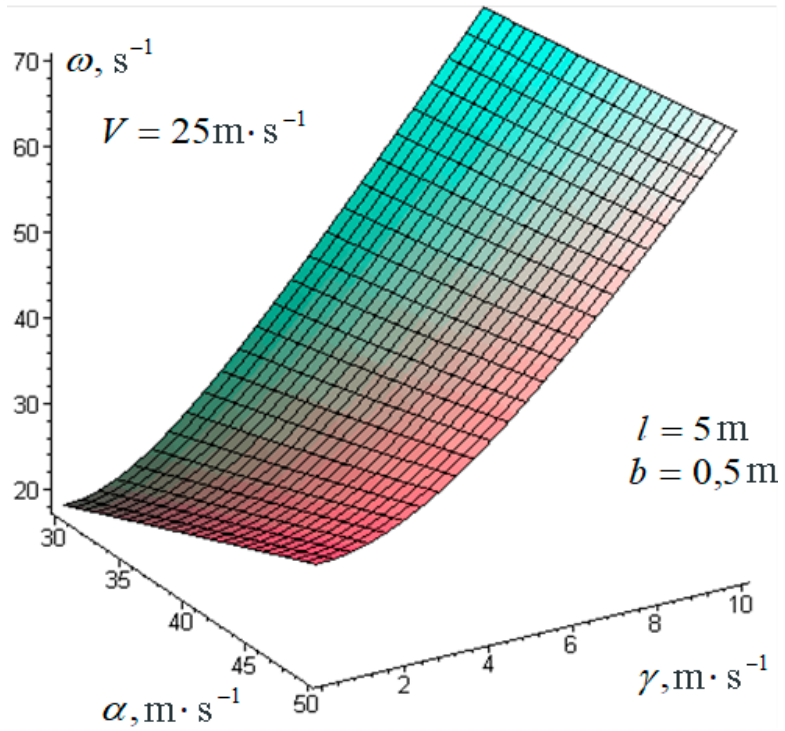

b

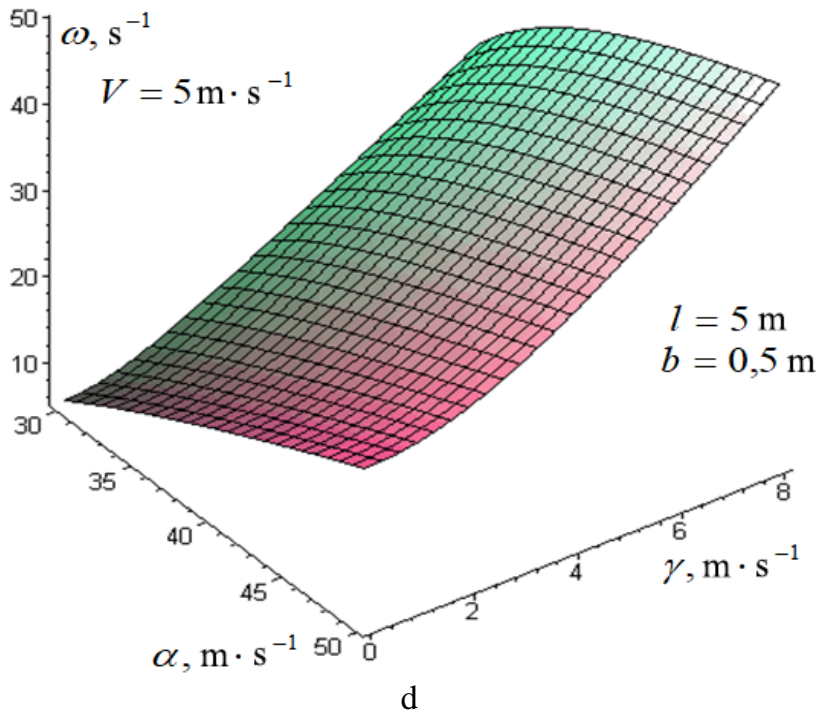

Fig. 1. The dependence of wave numbers on speed $V$ and physical-mechanical characteristics of environment (parameters $\alpha, \gamma$ ) and frequency of natural oscillations of these parameters 
Similarly, we find $u_{x t}, u_{x x}, u_{y y}$ :

$$
\begin{gathered}
u_{x t}(x, t)=-a \omega[\kappa \cos (\kappa x+\delta y+\theta)+\chi \cos (\chi x-\delta y-\theta)]- \\
-\varepsilon\left\{A_{1}(a)[\kappa \sin (\kappa x+\delta y+\theta)-\chi \sin (\chi x-\delta y-\theta)]-\right. \\
\left.-a B_{1}(a)[\kappa \cos (\kappa x+\delta y+\theta)+\chi \cos (\chi x-\delta y-\theta)]+\frac{\partial^{2} u_{1}}{\partial \theta \partial x} \omega\right\}+\varepsilon^{2} \ldots, \\
u_{x x}(x, t)=-a\left[\kappa^{2} \cos (\kappa x+\delta y+\theta)-\chi^{2} \cos (\chi x-\delta y-\theta)\right]+\varepsilon \frac{\partial^{2} u_{1}}{\partial x^{2}}+\varepsilon^{2} \ldots, \\
u_{y y}(x, t)=-a \delta^{2}[\cos (\kappa x+\delta y+\theta)-\cos (\chi x-\delta y-\theta)]+\varepsilon \frac{\partial^{2} u_{1}}{\partial y^{2}}+\varepsilon^{2} \ldots
\end{gathered}
$$

The asymptotic representation of the solution (11), (12) will satisfy the original equation (1), if after substituting in his place the function $u(x, y, t)$ and its derivative expressions that are consistent with (12)(15), coefficients of the same powers of a small parameter $\varepsilon$ of the right and left parts of it will be the same. The latter is a prerequisite for the determination of unknown functions $u_{1}(a, x, \theta), u_{2}(a, x, \theta), \ldots$, in particular, to find $u_{1}(a, x, \theta)$ let us obtain a linear differential equation:

$$
\begin{gathered}
\omega^{2} \frac{\partial^{2} u_{1}}{\partial \theta^{2}}+2 V \omega \frac{\partial^{2} u_{1}}{\partial \theta \partial x}-\left(\alpha^{2}-V^{2}\right) \frac{\partial^{2} u_{1}}{\partial x^{2}}-\gamma^{2} \frac{\partial^{2} u_{1}}{\partial y^{2}}=F_{1}(a, x, y \theta)+ \\
+2\left\{A_{1}(a)(\omega+\kappa V)(\sin (\kappa x+\delta y+\theta)+(\omega-\chi V) \sin (\chi x-\delta y-\theta))+\right. \\
\left.+a B_{1}(a)((\omega+\kappa V) \cos (\kappa x+\delta y+\theta)-(\omega-\chi V) \cos (\chi x-\delta y-\theta))\right\}, \\
F_{1}(a, x, \theta)=\left.f\left(u, u_{x}, u_{t}\right)\right|_{u=a(\cos (\kappa x+\delta y+\theta)-\cos (\chi x-\delta y-\theta)),} \\
u_{x}=-a(\kappa \sin (\kappa x+\delta y+\theta)-\chi \sin (\chi x-\delta y-\theta)), \\
u_{y}=-a \delta(\sin (\kappa x+\delta y+\theta)+\sin (\chi x-\delta y-\theta)), \\
u_{t}=-\alpha \omega(\sin (\kappa x+\delta y+\theta)+\sin (\chi x-\delta y-\theta)) .
\end{gathered}
$$

After simple transformations the coefficients of the $A_{1}(a)$ and $B_{1}(a)$ in the right side of equation (15) presented in the form:

$$
\begin{gathered}
{[(\omega+\kappa V) \sin (\kappa x+\delta y+\theta)+(\omega-\chi V) \sin (\chi x-\delta y-\theta)] A_{1}(a)+} \\
+a[(\omega+\kappa V) \cos (\kappa x+\delta y+\theta)-(\omega-\chi V) \cos (\chi x-\delta y-\theta)] B_{1}(a)= \\
=[(\omega+\kappa V) \sin (\kappa x+\delta y)+(\omega-\chi V) \sin (\chi x-\delta y)]\left(A_{1}(a) \cos \theta-a B_{1} \sin \theta\right)+ \\
+[(\omega+\kappa V) \cos (\kappa x+\delta y)-(\omega-\chi V) \cos (\chi x-\delta y)]\left(A_{1}(a) \sin \theta+a B_{1} \cos \theta\right) .
\end{gathered}
$$

For an unambiguous determination the unknown functions $A_{1}(a)$ and $B_{1}(a)$ from the differential equation (15) impose upon the function $U_{1}(a, x, y, \theta)$ additional conditions, namely: it can not contain the schedule of terms proportional $\sin \theta$ and $\cos \theta$. With $2 \pi$ periodicity of $\theta$ this function implies that the same properties are and its partial derivatives with respect to $\theta, x$ and $y$. This allows us to get from (17) the system of algebraic equations connecting the unknown function $A_{1}(a)$ and $B_{1}(a)$ in the form:

$$
\begin{gathered}
{[(\omega+\kappa V) \sin (\kappa x+\delta y)+(\omega-\chi V) \sin (\chi x-\delta y)] A_{1}(a)+} \\
+a[(\omega+\kappa V) \cos (\kappa x+\delta y)-(\omega-\chi V) \cos (\chi x-\delta y)] B_{1}(a)=\frac{-\varepsilon}{2 \pi} \int_{0}^{2 \pi} F_{1}(a, x, y, \theta) \cos d \theta
\end{gathered}
$$




$$
\begin{gathered}
{[(\omega+\kappa V) \cos (\kappa x+\delta y)-(\omega-\chi V) \cos (\chi x-\delta y)] A_{1}(a)-} \\
-a[(\omega+\kappa V) \sin (\kappa x+\delta y)+(\omega-\chi V) \sin (\chi x-\delta y)] B_{1}(a)=\frac{-\varepsilon}{2 \pi} \int_{0}^{2 \pi} F_{1}(a, x, y, \theta) \sin \theta d \theta .
\end{gathered}
$$

By averaging left and right parts of (18) by linear variables $x, y$, we find:

$$
\begin{gathered}
A_{1}(a)=\frac{\varepsilon}{2 \pi b l\left[(\omega+\kappa V)^{2}+(\omega-\chi V)^{2}\right]} \int_{0}^{l} \int_{0}^{b} \int_{0}^{2 \pi} f_{1}(a, x, y, \theta) \times \\
\times\{[(\omega+\kappa V) \sin (\kappa x+\delta y)+(\omega-\chi V) \sin (\chi x-\delta y)] \cos \theta+ \\
+[(\omega+\kappa V) \cos (\kappa x+\delta y)-(\omega-\chi V) \cos (\chi x-\delta y)] \sin \theta\} d \theta d y d x, \\
B_{1}(a)=\frac{\varepsilon}{a 2 b \pi l\left[(\omega+\kappa V)^{2}+(\omega-\chi V)^{2}\right]} \int_{0}^{l} \int_{0}^{b} \int_{0}^{2 \pi} f_{1}(a, x, \theta) \times \\
\times\{[(\omega+\kappa V) \sin (\kappa x+\delta y)+(\omega-\chi V) \sin (\chi x-\delta y)] \sin \theta- \\
-[(\omega+\kappa V) \cos (\kappa x+\delta y)-(\omega-\chi V) \cos (\chi x-\delta y)] \cos \theta\} d \theta d y d x .
\end{gathered}
$$

Defining functions that define the first approximation of the main characteristics of waves $A_{1}(a)$ and $B_{1}(a)$, we'll move to find functions $U_{1}(a, x, y, \theta)$.This function will satisfy the conditions imposed on it, defined in the form:

$$
U_{1}(a, x, y)=\sum_{k \neq 1} \sum_{m} \sum_{s} U_{1 k s m}(a) X_{s}\left(\frac{s \pi}{l} x\right) Y_{m}\left(\frac{m \pi}{b} y\right) \exp i(k \theta),
$$

where $\left\{X_{s}\left(\frac{s \pi}{l} x\right)\right\},\left\{Y_{m}\left(\frac{m \pi}{b} y\right)\right\}$ must complete orthogonal system of functions satisfy the appearance, namely $\left\{X_{s}\left(\frac{s \pi}{l} x\right)\right\}=\left\{\sin \frac{s \pi}{l} x\right\},\left\{Y_{m}\left(\frac{m \pi}{b} y\right)\right\}=\left\{\sin \frac{m \pi}{b} y\right\}$.

To find the unknown coefficients of expansion $U_{1 k s m}(a)$ from the differential equation (15) we have:

$$
\begin{gathered}
\sum_{k \sum \sum U_{i} U_{k s m}(a)}\left(\begin{array}{l}
\left.-k^{2} \omega^{2} X_{s}\left(\frac{s \pi}{l} x\right) Y_{m}\left(\frac{m \pi}{b} y\right)+2 i V \omega \frac{d X_{s}}{d x} Y_{m}\left(\frac{m \pi}{b} y\right)\right) \\
+\left(\left(\alpha^{2}-V^{2}\right)\left(\frac{s \pi}{l}\right)^{2}+\left(\frac{m \pi}{b}\right)^{2}\right) X_{s}\left(\frac{s \pi}{l} x\right) Y_{m}\left(\frac{m \pi}{b} y\right)
\end{array}\right) \cdot \exp (i(k \theta))= \\
=f(a, x, y, \theta)+2\left[\begin{array}{l}
(\omega+\kappa V) \sin (\kappa x+\delta y)+ \\
+(\omega-\chi V) \sin (\chi x-\delta \theta)
\end{array}\right]\left(A_{1}(a) \cos \theta-a B_{1} \sin \theta\right)+ \\
+2[(\omega+\kappa V) \cos (\kappa x+\delta y)-(\omega-\chi V) \cos (\chi x-\delta y)]\left(A_{1}(a) \sin \theta+a B_{1} \cos \theta\right) .
\end{gathered}
$$

Last value determines the unknown coefficients in the form:

$$
\begin{gathered}
U_{1 k s m}(a)=\frac{1}{\left(-k^{2} \omega^{2}+\left(\alpha^{2}-V^{2}\right)\left(\frac{s \pi}{l}\right)^{2}+\left(\frac{m \pi}{b}\right)^{2}\right) \pi l b} \int_{0}^{2 \pi} \int_{0}^{l} \int_{0}^{b} f_{1}(a, x, y, \theta) X_{s}\left(\frac{s \pi}{l} x\right) Y_{m}\left(\frac{m \pi}{b} y\right) \times \\
\times \exp (-i(k \theta)) d y d x d \theta, \quad k \neq 1 .
\end{gathered}
$$

Such species derived differential equations for the second and the following approximations, only the right side of the equation is a function $F_{2}(a, x, y, \theta)$ has more bulky look.

Below (in Fig. 2), for the case $f\left(u, u_{x}, u_{y}, u_{t}, u_{x x}, u_{y y}, u_{x y}\right)=K u_{t}+B u_{x x}^{3}$ there are presented graphical dependencies that illustrate the impact velocity of longitudinal motion, the amplitude and frequency parameters on natural oscillations. 


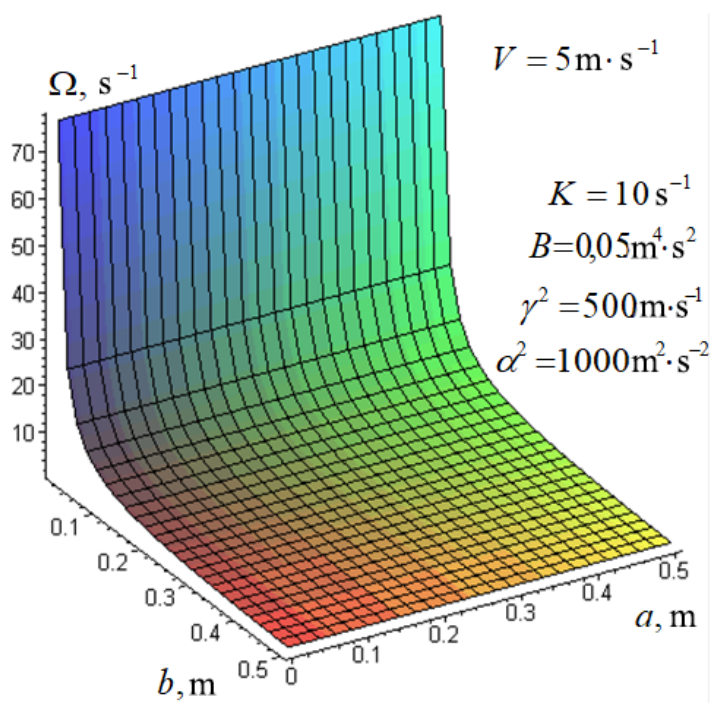

a

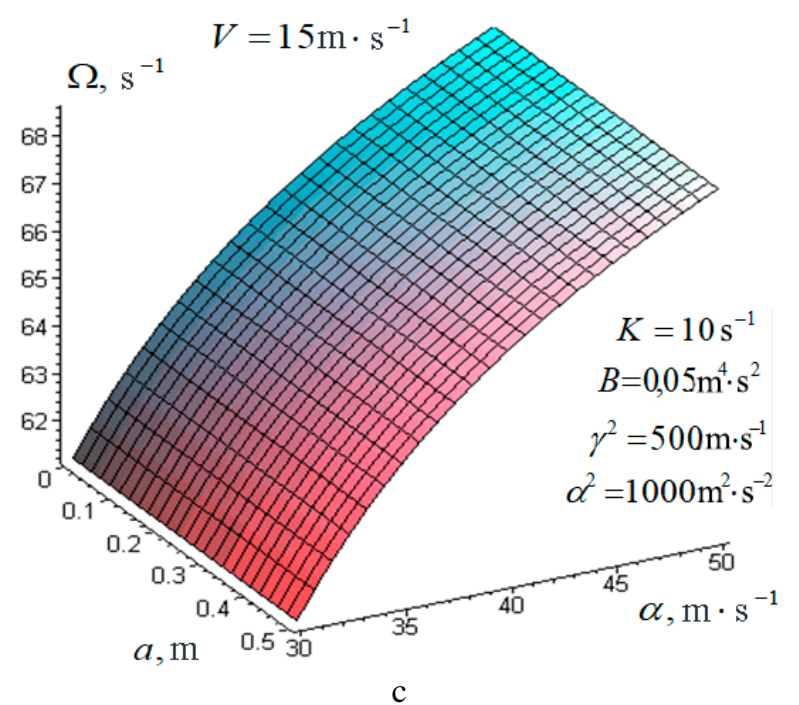

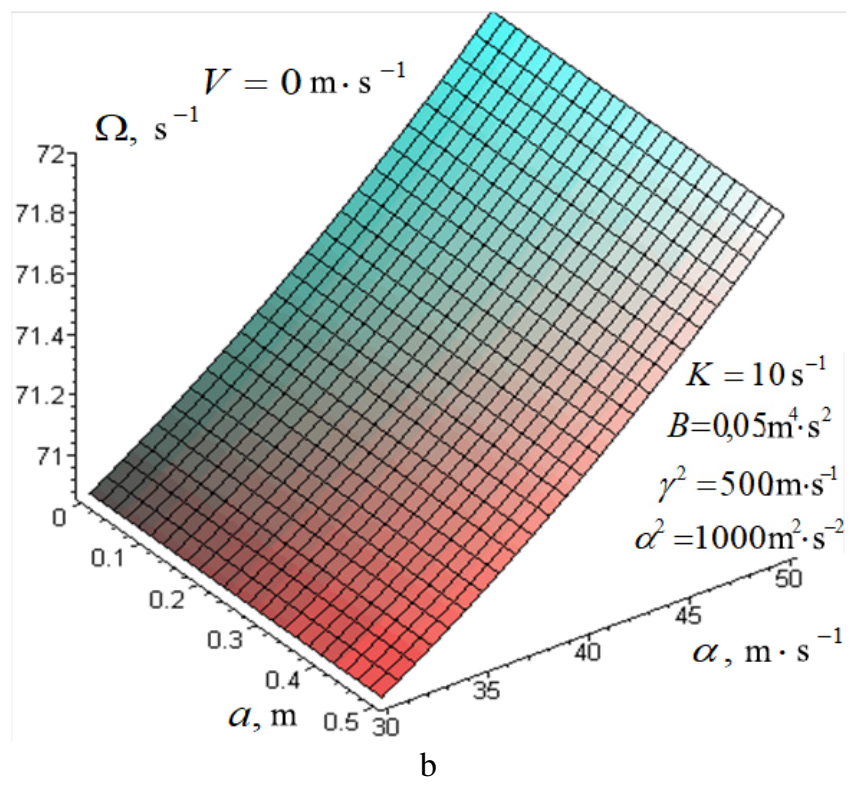

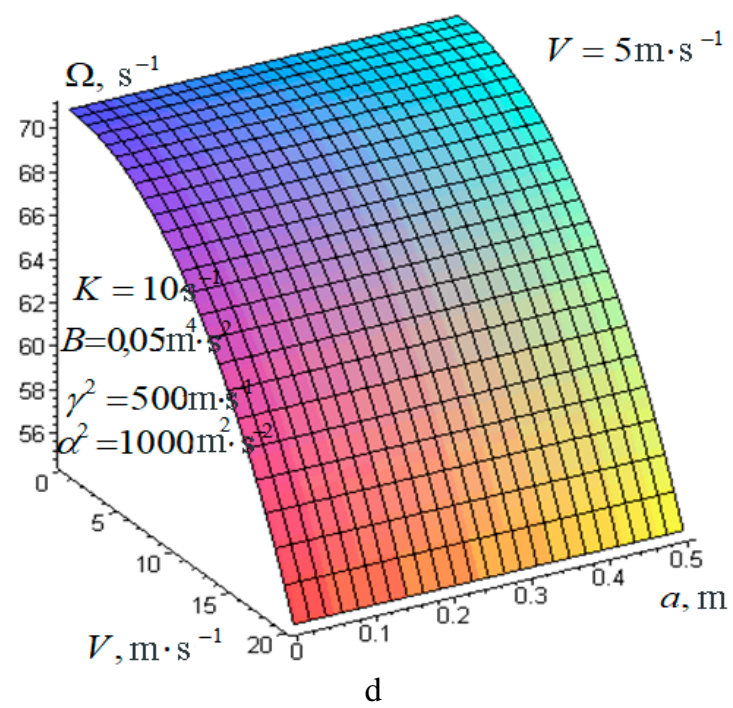

Fig. 2. The dependence of the oscillation frequency on the amplitude of the motion and the physical and mechanical characteristics of the environment at different speeds of longitudinal motion

\section{Conclusions}

Developed in work methodology allows to determine the impact velocity of longitudinal motion, different nature of nonlinear forces on the main parameters of oscillation of flexible elements of the systems gear and transportation, as well as loose environments in their vibratory processing. These formulas can serve as a basis for engineering calculations related elements of systems and presented on the basis of their image dependences recite a significant impact on both the physical and mechanical, geometrical parameters on the dynamics of the process, particularly for higher speeds longitudinal motion frequency natural oscillations is smaller and a value of the latter $\alpha$ is the failure of oscillation. The reliability of the developed method is confirmed by the fact that in the limiting case when $\gamma \rightarrow 0$. Based on the results value related to oscillation one-dimensional systems. The very same method will be the basis for a more complex case study of the dynamics longitudinally moving systems - external periodic perturbations.

\section{References}

[1] M. B. Sokil, O. L. Lyashuk, and A. P. Dovbush, "Dynamics of flexible elements of drive systems with variable contact point to the pulleys", INMATEH Agricultural Engineering, vol. 48, no. 1, pp. 119-124, 2016. 
[2] I. Gevko, O. Lyashuk, A. Djachun, and A. Dovbush, "Interpretation of the choice of conveyers with improved technological characteristics", MOTROL. Commission of Motorization and Energetics in Agriculture, vol. 17, no. 4, pp. 107-116, 2015.

[3] O. L. Lyashuk, et al., "Mathematical model of bending vibrations of a horizontal feeder-mixer along the flow of grain mixture", INMATEH Agricultural Engineering, vol. 55, no. 2, pp. 35-44, 2018.

[4] I. Hevko, et al., "Resonant oscillation of vertical working part of conveyer-loader", Bulletin of the Karaganda University. Physics series, no. 2 (94), pp. 73-82, 2019.

[5] Yu. A. Mitropolskii, and B. I. Moseenkov, Asimptoticheskie resheniia uravnenii v chastnykh proizvodnykh [Asymptotic solutions of partial differential equations]. Kyiv, Ukraine: Vyshcha shkola Publ., 1976. [in Russian].

[6] N. N. Bogoliubov, and Yu. A. Mitropolskii, Asimptoticheskie metody v teorii nelineinykh kolebanii [Asymptotic methods in the theory of nonlinear oscillations]. Moscow, Russia: Nauka Publ., 1974. [in Russian].

[7] I. Fazekas, and A. Kukush, "Asymptotic properties of estimators in nonlinear functional errors-in-variables with dependent error terms”, Journal of Mathematical Sciences, vol. 92, no. 3, pp. 3890-3895, 1998.

[8] Ye. V. Kharchenko, and M. B. Sokil, "Neliniyni protsesy u seredovyshchakh, yaki kharakteryzuyut'sya pozdovzhnim rukhom, i vplyv sposobu zakriplennya na yikh kolyvannya" ["Nonlinear processes in media characterized by longitudinal motion and the influence of the method of fixation on their oscillations"], Avtomatizaciâ virobničih procesìv u mašinobuduvannì ta priladobuduvannì [Industrial Process Automation in Engineering and Instrumentation], vol. 41, pp. 156-159, 2007. [in Ukrainian].

[9] Martyntsiv M. P., and Sokil M. B., “Odne uzahal'nennya metodu D'Alambera dlya system, yaki kharakteryzuyut'sya pozdovzhnim rukhom" ["One generalization of the D'Alembert method for systems characterized by longitudinal motion”], Naukovyy visnyk Derzhavnoho lisotekhnichnoho universytetu Ukrayiny [Scientific Bulletin of Ukrainian State Forestry University], vol. 13.4, pp. 64-67, 2003. [in Ukrainian].

[10] Y. A. Mitropol'skii, and B. I. Sokil, "On the application of Ateb-functions to the construction of an asymptotic solution of the perturbed nonlinear Klein-Gordon equation”, Ukrainian Mathematical Journal, no. 5 (50), pp. 754-760, 1998.

[11] B. I. Sokil, "On a method for constructing one-frequency solutions of a nonlinear wave equation", Ukrainian Mathematical Journal, no. 6 (46), pp. 853-856, 1994. 